\title{
WASTE TANK COOLING COIL LEAKAGE CALCULATIONS
} (U)

by L. O. Dworjanyn

Westinghouse Savannah River Company

Savannah River Site

Aiken, South Carolina 29808

Other Authors:

This paper was prepared in connection with work done under Contract No. DE-AC09-89SR18035 with the U. S. Department of Energy. By acceptance of this paper, the publisher and/or recipient acknowledges the U.S. Government's right to retain a nonexclusive, royalty-free license in and to any copyright covering this paper, along with the right to reproduce and to authorize others to reproduce all or part of the copyrighted paper. 


\section{DISCLAIMER}

This report was prepared as an account of work sponsored by an agency of the United States Government. Neither the United States Government nor any agency thereof, nor any of their employees, makes any warranty, express or implied, or assumes any legal liability or responsibility for the accuracy, completeness, or usefulness of any information, apparatus, product, or process disclosed, or represents that its use would not infringe privately owned rights. Reference herein to any specific commercial product, process, or service by trade name, trademark, manufacturer, or otherwise does not necessarily constitute or imply its endorsement, recommendation, or favoring by the United States Government or any agency thereof. The views and opinions of authors expressed herein do not necessarily state or reflect those of the United States Government or any agency thereof.

This report has been reproduced directly from the best available copy.

Available to DOE and DOE contractors from the Office of Scientific and Technical Information, P.O. Box 62, Oak Ridge, TN 37831; prices available from (615) 576-8401, FTS 626-8401.

Available to the public from the National Technical Information Service, U.S. Department of Commerce, 5285 Port Royal Rd., Springfield, VA 22161. 
Keywords:

High Level Waste,

Cooling Pipes, Waste

Tanks, Pipe Flow,

Orifice Discharge

Retention Time: Permanen $\imath$

December 20, 1991

cc: G.T. Wright, 703-H

R.W. Harrall, 703-H

P.D. d'Entremont, 703-H, (diskette attached)

O.M. Ebra-Lima, 773-A

K.W. Stevens, 773-A

L.M. Papouchado, 773-A

W.L. Tamosaitis, 773-A

SRL Records, 773-A (4)

TO: $\quad$ Brenda L. Lewis, 703-H

FROM: L. O. Dworjanyn, 779-2A

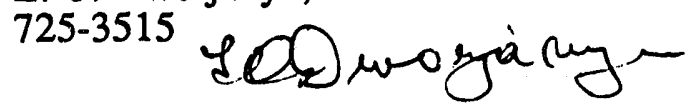

\section{WASTE TANK COOLING COIL LEAKAGE CALCULATIONS (U)}

A high activity Waste Tank cooling coil supply line cracked on September 12, 1991 at the H-Area East pump house, draining the cooling water to the ground. This raised the possibility of draining high-activity waste to the ground by siphon action through the submerged cooling coils since some of the cooling water supply lines are located up to $40 \mathrm{ft}$. below waste tank liquid level (Fig. 2). The following documentation summarizes conclusions and provides details of flow calculation presented earlier during the incident investigation.

1. No plausible reason for a simultaneous rupture of the supply line and the cooling coils inside the tank was identified. Both seismic stresses (Ref. ${ }^{4}$ ) and water hammer produce relatively low stresses on the cooling coils. A hypothetical simultaneous rupture of the cooling coils inside the tank and the supply line below ground could result in 100 to $200 \mathrm{gpm}$ waste discharge to the ground (Table 1).

2. Waste discharge from a possible cooling coil corrosion leak would be limited to $20 \mathrm{gal} / 12-\mathrm{hr}$ shift under maximum possible siphon, based on operating procedures for cooling water makeup which call for coil isolation when a cooling water loss of $40 \mathrm{gal} / \mathrm{shift}$ is identified (Fig 1). This level of discharge is within the existing envelope of accident consequences for Waste Tank Form SAR (Ref. ${ }^{5}$ ).

Details are attached, including corrections and comments by Paul d'Entremont. LOD/chc

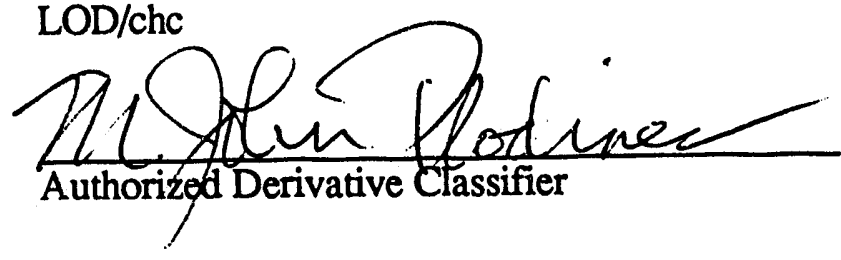




\section{A. WASTE TRANSFER VERIFICATION,}

Sample calculation for liquid waste transfer via siphon action.

Confirm reported $70 \mathrm{gpm}$ tank to tank transfer via a waste transfer line.

Assume: $2000 \mathrm{ft}$ long, $3 \mathrm{in}$. Schedule 40 steel pipe, $70 \mathrm{gpm}$ flow.

Calculate: Fluid head for assumed transfer. Use calculated factors in spreadsheel

Linear Fluid Velocity.

Internal pipe diameter, $d=3.068$ in.

$$
\text { Flow area, } \begin{aligned}
A & =\frac{\pi D^{2}}{4}=\frac{\pi \times d^{2}}{4 \times 144} \mathrm{ft}^{2} \\
& =\frac{\pi d^{2}}{576} \\
& =0.0513 \mathrm{ft}^{2}
\end{aligned}
$$

Volumetric flow, $Q=70 \mathrm{gpm}$

$$
\begin{aligned}
& =\frac{0.1337 \times 70}{60} \mathrm{ft}^{3} / \mathrm{sec} \\
& =0.002228 \times 70 \mathrm{ft}^{3} / \mathrm{sec} \\
& =0.1560 \mathrm{ft}^{3} / \mathrm{sec} \\
\text { Linear velocity, } V & =\frac{0.002228 Q}{A} \\
& =\frac{1.2833 Q}{\pi d^{2}} \\
& =\frac{1.2833 \times 70}{\pi \times 3.088^{2}} \\
& =3.038 \mathrm{ft} / \mathrm{sec}
\end{aligned}
$$

\section{Waste Flow Reynolds Number}

Internal pipe diameter, $d=3.068$ in.

Fluid density, $\rho=1.3 \mathrm{~g} / \mathrm{cc} \times 62.43$

$$
=81.16 \mathrm{lb} / \mathrm{ft}^{3}
$$

Fluid viscosity, $\mu=3.7 \mathrm{cp} / 1488$

$$
=0.00248 \mathrm{lb} / \mathrm{ft}-\mathrm{sec}
$$

Reynolds Number, $\operatorname{Re}=\frac{D v \rho}{\mu}$

$$
\begin{aligned}
& =\frac{3.068 \times 3.038 \times 81.16}{12 \times 0.002486} \\
& =25,350
\end{aligned}
$$




\section{Friction Factor}

Surface roughness, $\varepsilon=0.0018$ in. for steel pipe

Relative roughness, $\varepsilon / \mathrm{d}=0.0006$ for 3 in. pipe.

Fanning friction factor, $f=0.0064, \quad$ Ref. (1), 5-22

$=0.0066$ From spreadsheet correlation for 2 in. pipe

\section{Pressure Drop}

The pressure drop for steady flow in uniform circular pipes is given by the Fanning equation:

$$
\text { Friction loss in pipe, } \begin{aligned}
F & =\left(\frac{4 f L}{D}\right) \frac{V^{2}}{2 g_{c}}, \mathrm{ft} \text { of fluid flowing, } \operatorname{Ref}^{(1)}, 5-22 \\
\text { where } D & =\text { pipe diameter, } \mathrm{ft} \\
L & =\text { pipe length, } \mathrm{ft} \\
g_{c} & =\text { dimensional constant, } 32.17 \mathrm{lb}-\mathrm{ft} /(\mathrm{lb}-\text { force })\left(\mathrm{sec}^{2}\right) \\
& =\frac{4 \times 12}{2 \times 32.17} \times \frac{f L V^{2}}{d}, \text { where } d \text { is pipe diameter, in. } \\
& =0.746 \frac{f L V^{2}}{d} \\
& =0.746 \times \frac{0.0064 \times 2000 \times 3.032^{2}}{3.068} \\
& =28.7 \mathrm{ft} \text { of flowing fluid }
\end{aligned}
$$

\section{Conclusions:}

The calculated differential pressure head $(28.7 \times 1.3=37.3 \mathrm{ft}$ of water $)$ is consistent with typical tank liquid level elevation differences. 


\section{B. COOLING COIL SIPHON CALCULATIONS.}

Detailed calculations for possible cooling coil waste discharge are shown in attached spreadsheet (Table 1), assuming:

(1) A completely submerged line break inside the waste tank.

(2) A submerged line break outside the tank allowing siphon suction.

(3) Maximum suction of one atmosphere (14.7 psi, or $26.1 \mathrm{ft}$ liquid waste).

The friction factor (Col B) was obtained from Ref.(1) and correlated with the Reynolds number using a third power polynomial ( $\mathrm{Col} \mathrm{G}$ ) to permit automatic spreadsheet calculations. A higher order polynomial $(\mathrm{Col} \mathrm{H})$ offered little improvement in the correlation. The resultant calculations for different pipe diameters (Col A), flows (Col B), pipe length (Col F) and resultant pressure drop (Col G) are shown in Table 1. For the 2 in. cooling coil line the equations were solved for flow corresponding to $1 \mathrm{~atm}$ or $26 \mathrm{ft}$ liquid waste differential pressure. The maximum physical differential height between waste tank liquid surface level and the lowest possible cooling coil location is about $31 \mathrm{ft}$. This would result in a correspondingly higher flow. The length of the two inch cooling line (Col F) would depend on the location of a hypothetical break, with a minimum of about $30 \mathrm{ft}$. to the first submersion point. Pressure drops in the 6 in. and 8 in. supply lines are relatively small, but would tend to reduce the flow.

\section{ORIFICE DISCHARGE CALCULATIONS.}

1. Waste leakage from waste tank into a cooling coil under siphon suction.

Assume: $\quad(1 / 32)$ in. corrosion orifice.

Maximum atmospheric siphon, $33.9 \mathrm{ft}$ water.

Waste density $1.3 \mathrm{~g} / \mathrm{cc}$.

The volumetric discharge is given by:

$$
Q=C A \sqrt{\frac{2 g_{c} \Delta P}{\rho\left(1-\beta^{4}\right)}} \operatorname{Ref}^{(6)}
$$

where $Q=$ flow, $\mathrm{ft}^{3} /$ ses

$C=$ orifice discharge coefficient, assume 1.0

$A=$ orifice area, $\mathrm{ft}^{2}$

$g_{c}=32.17 \mathrm{lb}-\mathrm{ft} /(\mathrm{lb}-$ force $)\left(\sec ^{2}\right)$

$\Delta P=$ differential pressure across the orifice, $\mathrm{lb} / \mathrm{ft}^{2}$

$\rho=$ discharge liquid density, $\mathrm{lb} / \mathrm{ft}^{3}$

$\beta=$ diameter ratio, assumed zero 


$$
\begin{aligned}
Q & =\frac{\pi d^{2}}{4 \times 144} \sqrt{2 \times 32.17} \sqrt{\frac{\Delta P}{\rho}} \quad \text { where } \mathrm{d}=\text { diameter, in. } \\
& =0.04375 d^{2} \sqrt{\frac{14.7 \times 144}{81.16}} \\
& =0.2234 d^{2} \mathrm{ft}^{3} / \mathrm{sec} \times 448.8 \\
& =100.28 d^{2} \mathrm{gpm} \\
& =100.27\left(\frac{1}{32}\right)^{2} \\
& =0.0979 \mathrm{gpm} \\
& =141 \mathrm{gal} / \mathrm{day}
\end{aligned}
$$

2. Cooling water leakage into a waste tank.

Assume: (1/32) in. corrosion orifice.

Cooling water pressure 90 psi.

Cooling water density $1.0 \mathrm{~g} / \mathrm{cc}$.

The volumetric discharge is given by:

$$
\begin{aligned}
Q & =C A \sqrt{\frac{2 g_{c} \Delta P}{\rho}} \quad \text { Ref }{ }^{(1)} 5-11 \\
& =0.04375 d^{2} \sqrt{\frac{90 \times 144}{62.43}} \\
& =0.6303 d^{2} \mathrm{ft}^{3} / \mathrm{sec} \times 448.8 \\
& =282.9 d^{2} \mathrm{gpm} \\
& =282.9\left(\frac{1}{32}\right)^{2} \\
& =0.276 \mathrm{gpm} \\
& =398 \mathrm{gal} / \text { day }
\end{aligned}
$$




\section{WATER HAMMER CALCULATIONS}

Assume: 90 psi maximum header pressure.

$800 \mathrm{ft}$ minimum coil length.

Cooling water flow is stopped instently.

Schedule 40 steel pipe, $\mathrm{OD}=2.375$, ID $=2.067$.

Calculate: Maximum linear velocity inside the cooling coil.

Maximum pressure surge due to water hammer.

Calculated linear velocity $=9.73 \mathrm{ft} / \mathrm{sec} @ 90 \mathrm{psi}, \quad$ Table 1

$$
\begin{aligned}
\text { Water hammer, } \mathrm{H}_{\mathrm{wh}} & =\frac{C \Delta V}{g_{c}} \mathrm{ft}, \quad \text { Ref. }{ }^{(2)} \mathrm{p} 1016 \\
\text { where } C & =\text { velocity of sound, } \mathrm{ft} / \mathrm{sec} \\
\Delta V & =\text { liquid velocity change, } \mathrm{ft} / \mathrm{sec} \\
g_{c} & =\text { gravitational constant } \\
\text { Therefore, } \mathrm{H}_{\mathrm{wh}} & =\frac{4820 \times 9.73}{32.17} \\
& =1458 \mathrm{ft} / 33.9 \\
& =43.0 \mathrm{~atm} \times 14.7 \\
& =632 \mathrm{psi} \text { pressure rise. } \\
\text { Maximum hoop stress } & =\frac{\text { Force }}{\text { area }} \\
& =\frac{(632+90) \times 2.067}{0.308} \\
& =4,846 \mathrm{psi}
\end{aligned}
$$

vs 30,000 psi steel pipe yield strength, or 48,000 psi tensile strength.

\section{E.REFERENCES}

(1) Perry and Chilton, "Chemical Engineer's Handbook", $5^{\text {th }}$ Edition.

(2) Cheremisinoff, "Encyclopedia of Fluid Mechanics", Vol.2, p 1016.

(3) Clarke \& Davidson, "Manual for Process Engineering Calculations", 2nd Edition.

(4) W.W.F. Yau, Memo to E.W. Holtzchider, "Seismic Resistance of the Cooling Coils for Type II and III Waste Tanks", WSRC-RP-91-937, Sep 18, 1991.

(5) W.C. Perkins and C.R. Lux, "Cooling-Water Releases in Waste Tank Farm", WSRC-TR-91-562.

(6) Douglass M. Considine, "Process Instruments and Controls Handbook", $3^{\text {nd }}$ Edition 


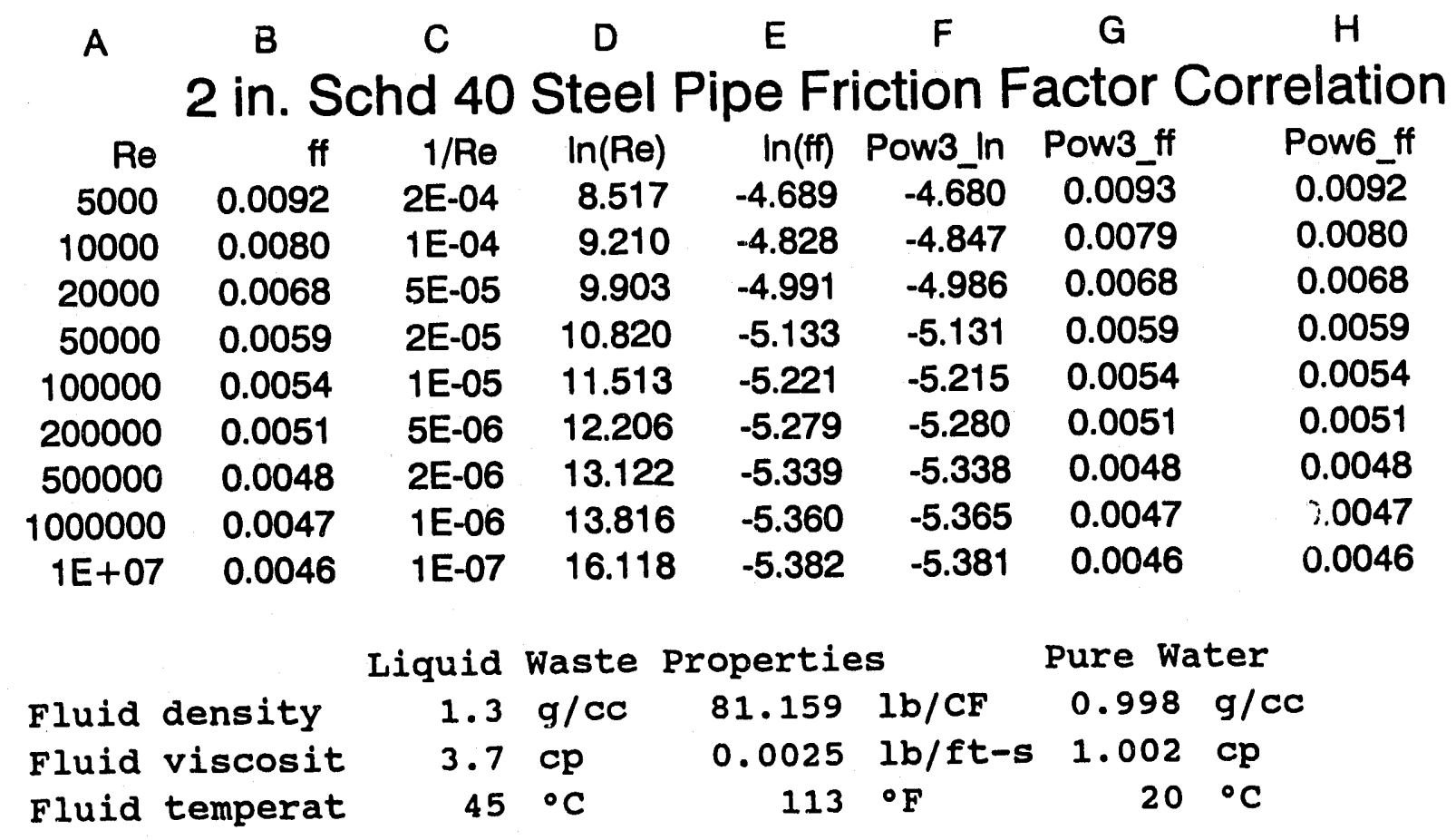

3 in. Transfer Line Discharge via Siphon Action

$\begin{array}{rrrrrrr}\begin{array}{r}\text { Pipe ID } \\ \text { in. }\end{array} & \begin{array}{r}\text { Flow } \\ \text { gpm }\end{array} & \begin{array}{r}\text { Vel. } \\ \mathrm{ft} / \mathrm{sec}\end{array} & \begin{array}{r}\text { ReyNo } \\ \text { Re }\end{array} & \begin{array}{r}\text { Fanning } \\ \text { ff }\end{array} & \begin{array}{r}\text { Pipe Len } \\ \mathrm{ft}\end{array} & \begin{array}{r}\text { PrDrop } \\ \mathrm{ft} \\ \text { CALC }\end{array} \\ 3.068 & 70 & 3.04 & 25350 & 0.0064 & 2000 & 28.72 \\ 3.068 & 65.54 & 2.84 & 23734 & 0.0066 & 2000 & 26.08 \equiv \text { Atm Pres. }\end{array}$

\section{2 in. Cooling Coil Siphon Discharge}

$\begin{array}{rrrrrrll}2.067 & 22.94 & 2.19 & 12331 & 0.0075 & 2000 & 26.08 & \\ 2.067 & 49.30 & 4.71 & 26498 & 0.0065 & 500 & 26.08 & \\ 2.067 & 78.91 & 7.54 & 42414 & 0.0060 & 210 & 26.08 & 3 \text { Loops } \\ 2.067 & 124.25 & 11.88 & 66788 & 0.0057 & 90 & 26.08 & 1 \text { Loop } \\ 2.067 & 222.28 & 21.25 & 119480 & 0.0053 & 30 & 26.08 & \text { Min length }\end{array}$

Max flow in $800 \mathrm{ft}$ cooling coil at 90 psi pressure
$2.067 \quad 101.81$
9.73
54724
0.0058
$800 \quad 159.67 \equiv 90$ psig

Field Supply Lines Pressure Drop

$\begin{array}{rrrrrrrr}6.065 & 100 & 1.11 & 18320 & 0.0069 & 1000 & 1.05 & 0.0003 \\ 6.065 & 1000 & 11.11 & 183195 & 0.0044 & 100 & 6.67 & 0.0003 \\ 7.981 & 100 & 0.64 & 13922 & 0.0071 & 1000 & 0.27 & 0.0002 \\ 7.981 & 1000 & 6.41 & 139215 & 0.0045 & 100 & 1.73 & 0.0002\end{array}$



A
B
C
D
E
$F$
G
H

\section{Orifice Flow Calculations}

Discharge at 1 atm liquid siphon

$\begin{array}{rrrrrrrr}\begin{array}{r}\text { Orific ID } \\ \text { in. }\end{array} & \begin{array}{r}\text { Flow } \\ \text { gpm }\end{array} & \begin{array}{r}\text { Orif Vel. } \\ \text { ft/sec }\end{array} & \begin{array}{r}\text { ReyNo } \\ \text { Re }\end{array} & \begin{array}{r}\text { Gallons/ } \\ \text { day }\end{array} & \begin{array}{r}\text { Flow } \\ \text { gpm }\end{array} & \begin{array}{r}\text { Gallons/ } \\ \text { day }\end{array} \\ 0.125 & 1.567 & 40.96 & 13926 & 2256 & 4.420 & 6365 & 1 / 8 \text { in. } \\ 0.0625 & 0.392 & 40.96 & 6963 & 564 & 1.105 & 1591 & 1 / 16 \text { in. } \\ 0.03125 & 0.098 & 40.96 & 3481 & 141 & 0.276 & 398 & 1 / 32 \text { in. } \\ & & & & & & & \\ & & & & & & & \\ 0.001 & 0.000 & 40.96 & 111 & 0 & 0.000 & 0 & \\ 0.002 & 0.000 & 40.96 & 223 & 1 & 0.001 & 2 & \\ 0.005 & 0.003 & 40.96 & 557 & 4 & 0.007 & 10 & \\ 0.010 & 0.010 & 40.96 & 1114 & 14 & 0.028 & 41 & \\ 0.015 & 0.023 & 40.96 & 1671 & 32 & 0.064 & 92 & \\ 0.020 & 0.040 & 40.96 & 2228 & 58 & 0.113 & 163 & \\ 0.025 & 0.063 & 40.96 & 2785 & 90 & 0.177 & 255 & \\ 0.03125 & 0.098 & 40.96 & 3481 & 141 & 0.276 & 398 & 1 / 32 \text { in. } \\ 0.040 & 0.130 & 40.96 & 4456 & 231 & 0.453 & 652 & \\ 0.050 & 0.251 & 40.96 & 5570 & 361 & 0.707 & 1018 & \\ 0.060 & 0.361 & 40.96 & 6684 & 520 & 1.018 & 1467 & \\ 0.070 & 0.491 & 40.96 & 7799 & 708 & 1.386 & 1996 & \\ 0.080 & 0.642 & 40.96 & 8913 & 924 & 1.811 & 2607 & \\ 0.090 & 0.812 & 40.96 & 10027 & 1170 & 2.291 & 3300 & \\ 0.100 & 1.003 & 40.96 & 11141 & 1444 & 2.829 & 4074 & \\ 0.110 & 1.213 & 40.96 & 12255 & 1747 & 3.423 & 4929 & \\ 0.120 & 1.444 & 40.96 & 13369 & 2079 & 4.074 & 5866 & \\ 0.130 & 1.695 & 40.96 & 14483 & 2440 & 4.781 & 6885 \\ 0.140 & 1.965 & 40.96 & 15597 & 2830 & 5.545 & 7985 & \\ 0.150 & 2.256 & 40.96 & 16711 & 3249 & 6.365 & 9166 \\ 0.160 & 2.567 & 40.96 & 17825 & 3696 & 7.242 & 10429 \\ 0.170 & 2.898 & 40.96 & 18939 & 4173 & 8.176 & 11773 & \\ 0.180 & 3.249 & 40.96 & 20053 & 4678 & 9.166 & 13199 & \\ 0.190 & 3.620 & 40.96 & 21167 & 5212 & 10.213 & 14706 & \\ 0.200 & 4.011 & 40.96 & 22282 & 5776 & 11.316 & 16295 & \\ & & & & & & & \end{array}$


FIGURE 1

\section{Corrosion Leak Impact 90 psi Chromated Water, 1 Atm. Syphon}

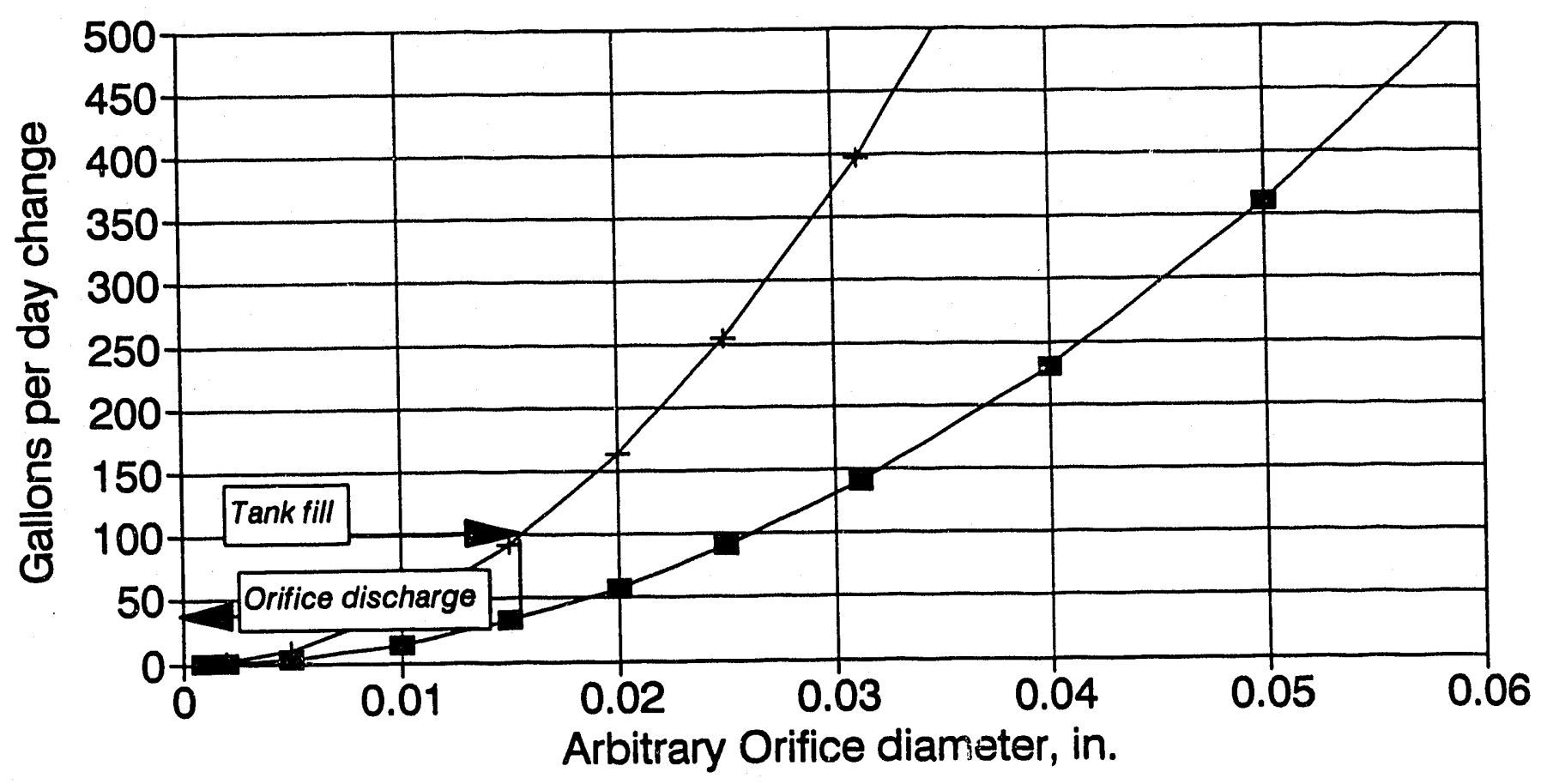

$\rightarrow-$ Orifice Discharge — Tank Fill 


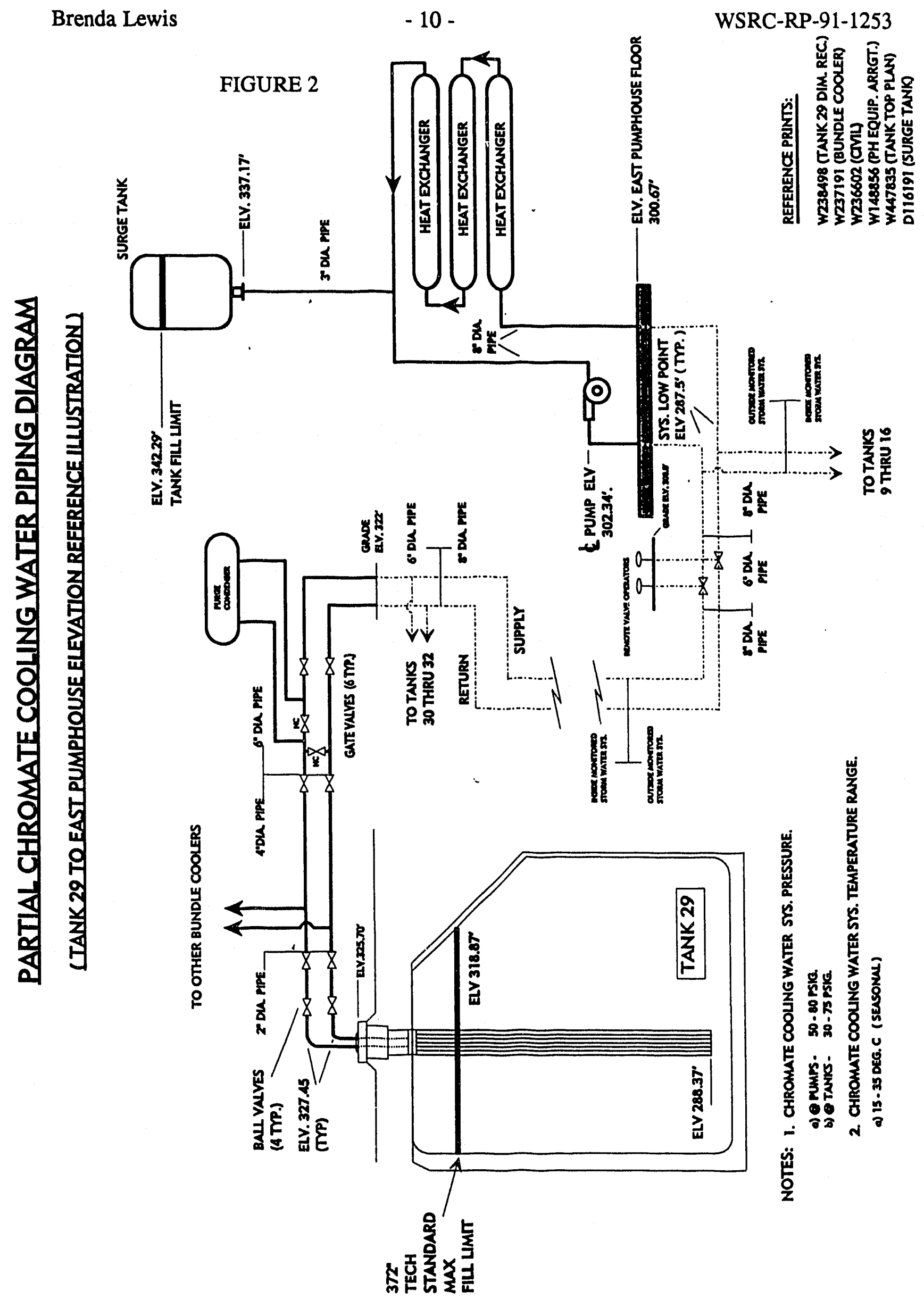



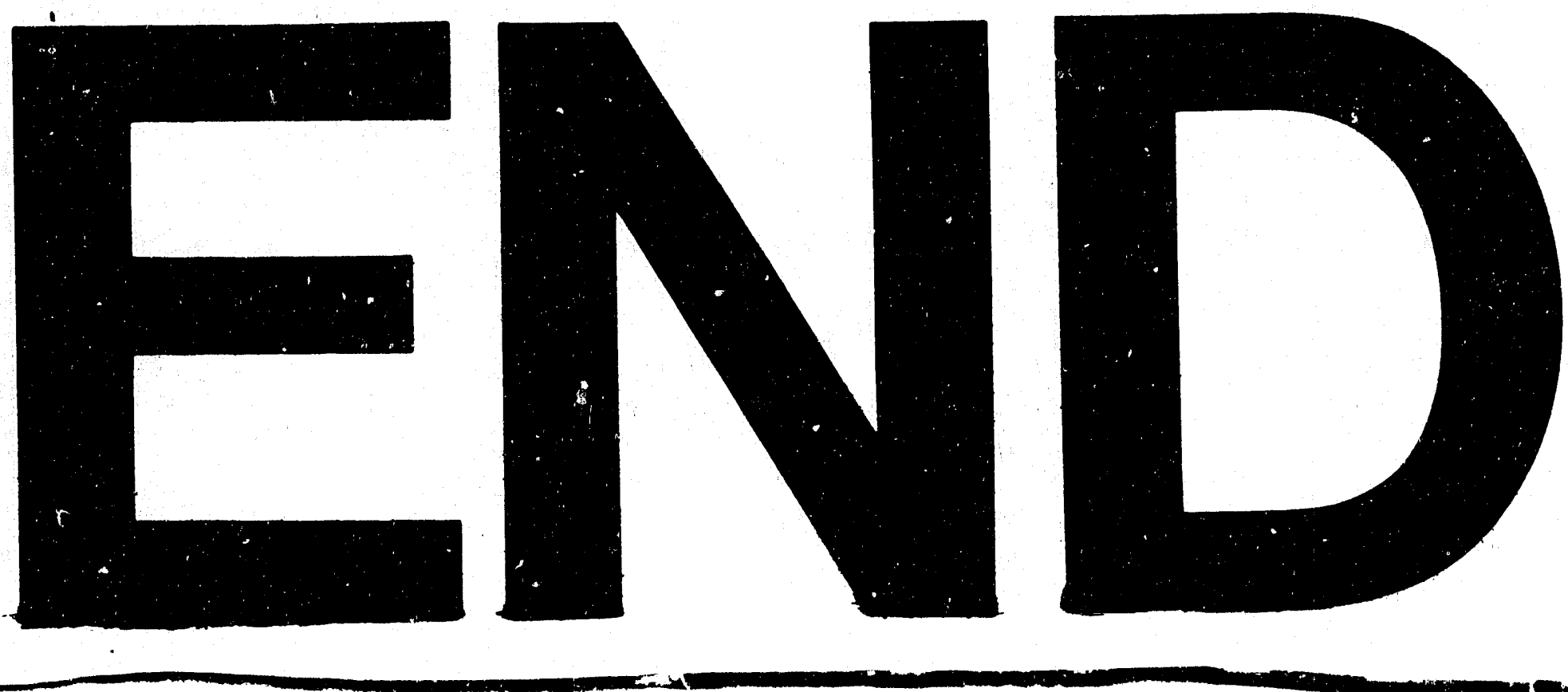

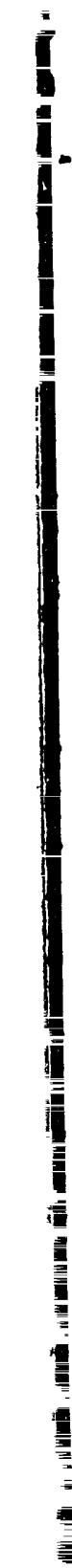
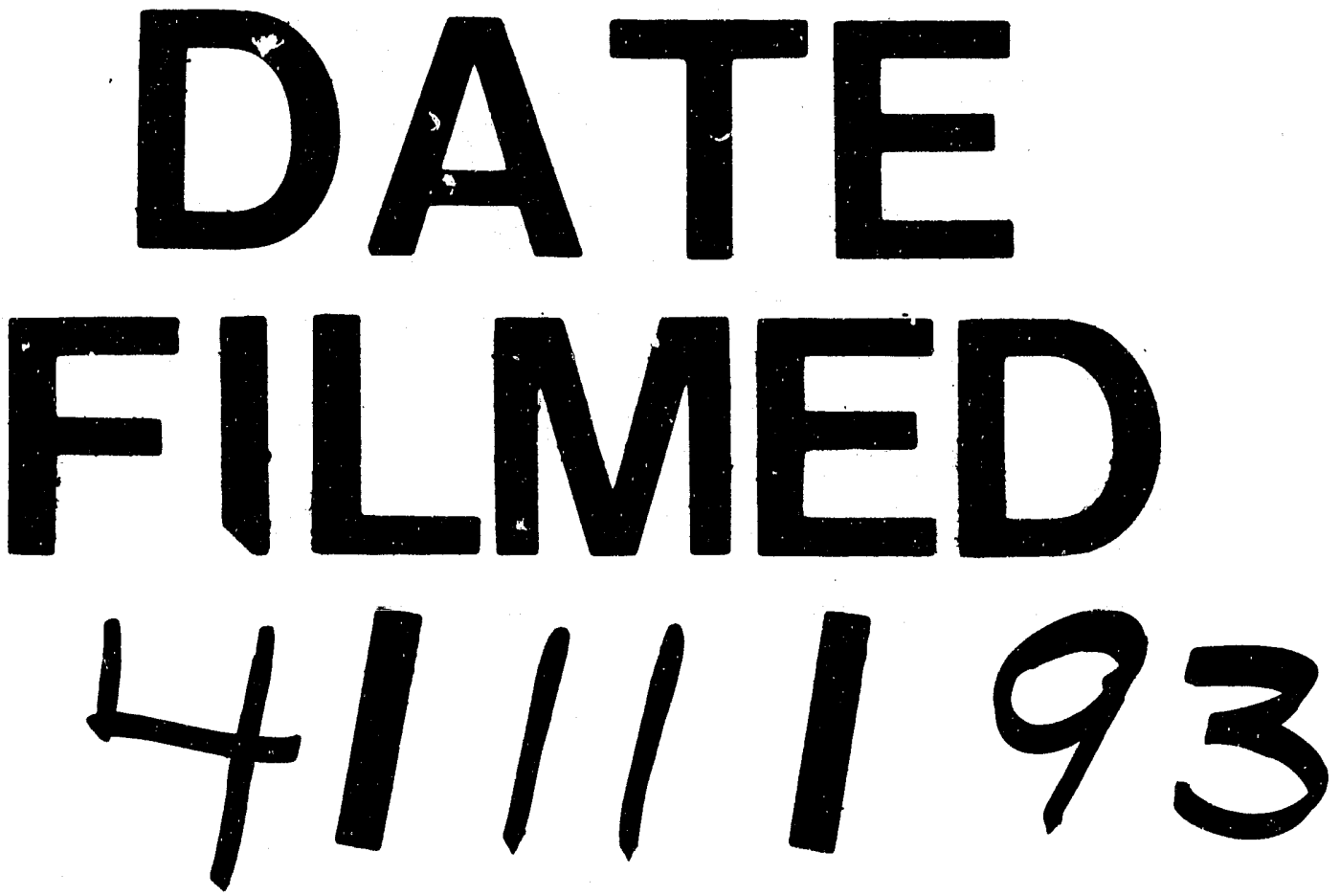
